\title{
Design of a Data-Oriented Kansei Feedback Control System
}

\author{
Takuya Kinoshita \\ Graduate School of Engineering, Hirosihma University, \\ 1-4-1, Kagamiyama, Higashihiroshima city, Hiroshima, Japan
}

Toru Yamamoto

Faculty of Engineering Division of Electrical, Systems and Mathematical Engineering, Hirosihma University, 1-4-1, Kagamiyama, Higashihiroshima city, Hiroshima, Japan

E-mail: takuya-kinoshita@hiroshima-u.ac.jp,yama@hiroshima-u.ac.jp

http://www.hiroshima-u.ac.jp

\begin{abstract}
In the development of the aging society, it is important for patients with hemiplegia to introduce adaptive welfare equipment. However, it is difficult to determine the suitable reference signal for each person. In this study, the design of a data-oriented cascade control system based on Kansei is proposed. In the proposed control system, there are two controllers which are a data-driven controller and a fixed controller. In particular, a data-driven controller determines the suitable reference signal based on Kansei.
\end{abstract}

Keywords: PID controller, Data-driven controller, Kansei, off-line learning.

\section{Introduction}

In the development of aging society, it is important for patients with hemiplegia to introduce the adaptive welfare equipment. However, it is difficult to support them by using general welfare equipment because there are a lot of individual disabilities. Therefore, an adaptive welfare equipment is required in near future. Nevertheless, it is difficult to determine the suitable reference signal for each person. In this study, the design of a data-oriented cascade control system based on Kansei is proposed. In the proposed control system, there are two controllers which are a data-driven controller $^{1}$ and a fixed controller. In particular, a data-driven controller ${ }^{1}$ determines the suitable reference signal of a welfare equipment based on Kansei.

\section{Schematic figure of the proposed control system}

Fig. 1 shows the schematic figure of the proposed control system. It is difficult for the patient with hemiplegia to move their foot by only torque $\tau_{B}$. Hence, in the proposed scheme, the Ankle Foot Orthosis ${ }^{2}$ (AFO) supports torque $\tau_{A}$. In this paper, Kansei signal is defined as walking comfortable $\mathrm{y}(t)$ whose maximum value is 1 . Note that it is important to estimate reference signal of brain $r_{\theta_{1}}(t)$ because $r_{\theta_{1}}(t)$ is unknown. Therefore, a data-base controller (primary controller) is applied to calculate the estimated reference signal $\mathrm{w}(t)$.

\section{Controlled object}

Fig. 2 shows the schematic figure of tow vertical joint manipulator is shown as leg model. $I_{1}, I_{2}$ are ankle's and knee's moment of inertia, respectively. $m_{0}, m_{1}, m_{2}$ are weight of upper body, lower leg and femur, respectively. $L_{1}, L_{2}$ are the length of lower leg and femur, and $l_{1}, l_{2}$ are the length of gravitational center of lower leg and femur, respectively. The torque $\tau_{1}$ and $\tau_{2}$ are corresponding to angle $\theta_{1}$ and $\theta_{2}$. The equation of walking motion is expressed as follows:

$$
\begin{aligned}
& {\left[\begin{array}{cc}
I_{1}+I_{2}+m_{0} L_{1}^{2}+2 m_{02} L_{1} l_{2} C\left(\theta_{2}\right) & I_{2}+m_{02} L_{1} l_{2} C\left(\theta_{2}\right) \\
I_{2}+m_{02} L_{1} l_{2} C\left(\theta_{2}\right) & I_{2}
\end{array}\right]\left[\begin{array}{c}
\ddot{\theta}_{1} \\
\ddot{\theta_{2}}
\end{array}\right]} \\
& +\left[\begin{array}{c}
-m_{02} L_{1} l_{2} C\left(\theta_{2}\right) \cdot\left(2 \dot{\theta}_{1} \dot{\theta_{2}}+\dot{\theta_{2}^{2}}\right) \\
m_{02} L_{1} l_{2} C\left(\theta_{2}\right) \cdot \dot{\theta_{1}^{2}}
\end{array}\right]
\end{aligned}
$$




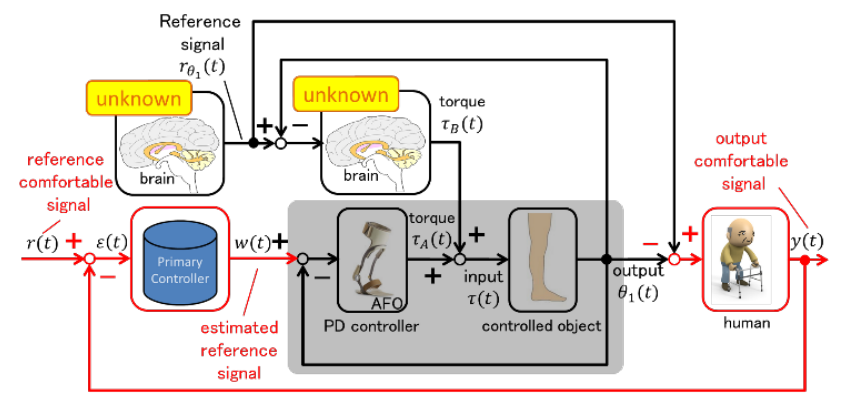

Fig. 1. Schematic figure of the proposed control system.

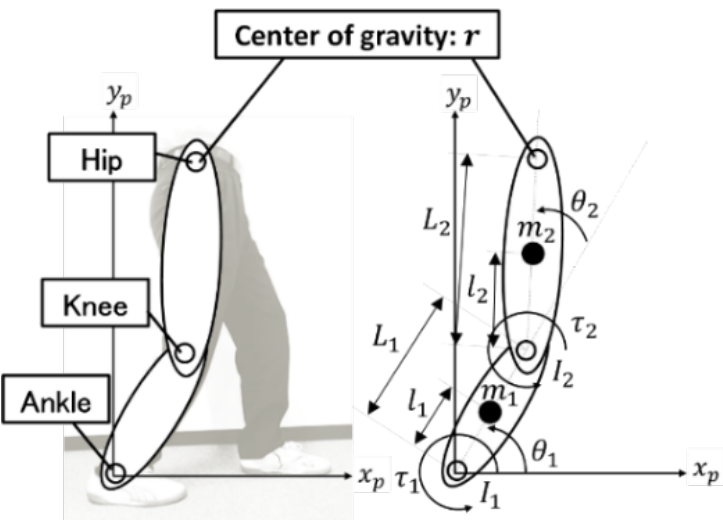

Fig. 2. Leg model: two vertical joint manipulators.

$$
+\left[\begin{array}{c}
\left(m_{1} l_{1}+m_{02} L_{1}\right) C\left(\theta_{1}\right)+m_{02} l_{2} C\left(\theta_{1}+\theta_{2}\right) \\
m_{02} l_{2} C\left(\theta_{1}+\theta_{2}\right)
\end{array}\right] g=\left[\begin{array}{l}
\tau_{1} \\
\tau_{2}
\end{array}\right]
$$

where $C(\theta)$ and $m_{02}$ denote $C(\theta)=\cos \theta$ and $m_{02}=m_{0}+m_{2}$, respectively.

In addition, Kansei signal $\mathrm{y}(t)$ in Fig. 1 is expressed as the following equation based on Weber-Fechner law ${ }^{3}$ :

$$
\begin{gathered}
y(t)=\frac{1}{1+\log \left(1+e_{\theta}(t)\right)} \\
e_{\theta}(t)=r_{\theta_{1}}(t)-\theta_{1}(t),
\end{gathered}
$$

where $r_{\theta_{1}}(t)$ is reference signal of $\theta_{1}(t)$.

\section{Design of a data-driven controller in outer loop as primary controller}

\subsection{Control law of a primary PID controller}

The primary controller in Fig. 1 is designed as a data-driven controller ${ }^{1}$ because the Kansei is nonlinear in equation (2). The primary controller is defined as follows:

$$
\begin{aligned}
& \Delta w(t)=K_{I} \varepsilon(t)-K_{P} \Delta y(t)-K_{D} \Delta^{2} y(t) \\
& \varepsilon(t):=r(t)-y(t),
\end{aligned}
$$

where $K_{P}, K_{I}$ and $K_{D}$ are proportional gain, integral gain and derivative gain, respectively. $\Delta$ denotes a difference operator. Note that the controller of AFO in

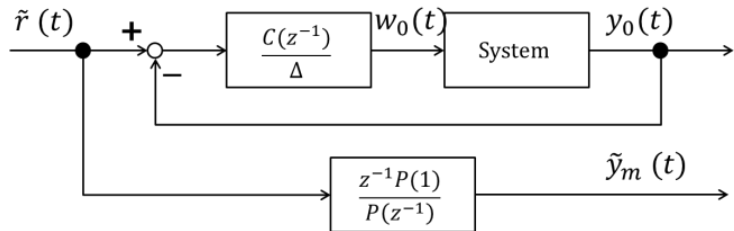

Fig. 3. Block diagram of the FRIT.

inner loop is designed as the following fixed PD controller.

$$
\begin{gathered}
\tau_{A}(t)=K_{P 2} e(t)+K_{D 2} \Delta e(t) \\
e(t):=w(t)-\theta_{1}(t),
\end{gathered}
$$

where $K_{P 2}$ and $K_{D 2}$ respectively are proportional gain and derivative gain.

\subsection{Design procedure of a data-driven control}

[STEP 1] Create an initial database.

The database is constructed by the following information vector:

$$
\begin{gathered}
\boldsymbol{\phi}(j):=[\overline{\boldsymbol{\phi}}(j), \boldsymbol{K}(j)] \quad(j=1,2, \cdots, N) \\
\overline{\boldsymbol{\phi}}(j):=\left[r(t+1), r(t), y(t), \cdots, y\left(t-n_{y}+1\right),\right. \\
\left.w(t-1), \cdots, w\left(t-n_{w}\right)\right] \\
\boldsymbol{K}(j):=\left[K_{P}(t), K_{I}(t), K_{D}(t)\right],
\end{gathered}
$$

where $N$ denotes the number of data.

[STEP 2] Calculate distance and select neighbors' data The distance between query $\bar{\phi}(t)$ and $\bar{\phi}(j)$ is calculated by using the following $\mathcal{L}_{1-\text { norm: }}$

$$
d(\overline{\boldsymbol{\phi}}(t), \overline{\boldsymbol{\phi}}(j))=\sum_{l=1}^{n_{y}+n_{w}+1}\left|\frac{\overline{\boldsymbol{\phi}}_{l}(t)-\overline{\boldsymbol{\phi}}_{l}(j)}{\max \overline{\boldsymbol{\phi}}_{l}(m)-\min \overline{\boldsymbol{\phi}}_{l}(m)}\right| .(11)
$$

$\overline{\boldsymbol{\phi}}_{l}(j)$ denotes the $l$ th element of query $\overline{\boldsymbol{\phi}}(j)$. $\max \overline{\boldsymbol{\phi}}_{l}(m)$ is a maximum value of the $l$ th element in database. In contrast, $\min \overline{\boldsymbol{\phi}}_{l}(m)$ is a minimum value of the $l$ th element. In addition, the number of neighbors' data $k$ are selected, which data are based on smallest distance value $d$.

[STEP 3] Calculate control parameters.

Control parameters are calculated by using the following Linearly Weighted Average (LWA)

$$
\boldsymbol{K}(\mathrm{t})=\sum_{i=1}^{k} w_{i} \boldsymbol{K}(i), \sum_{i=1}^{k} w_{i}=1,
$$

where $w_{i}$ is the weight parameter corresponding to the $i$ th information vector $\bar{\phi}(i)$ in the selected neighbors' data. It is calculated by the following equation:

$$
w_{i}=\frac{1 /\left(1+d_{i}\right)}{\sum_{i=1}^{k}\left\{1 /\left(1+d_{i}\right)\right\}} .
$$

The off-line learning method is described in next section. 


\subsection{Fictitious Reference Iterative Tuning: FRIT}

Fig. 3 shows a block diagram of the FRIT ${ }^{4}$. FRIT is a scheme to calculate control parameters directly from closed-loop data which are input $w_{0}(t)$, output $y_{0}(t)$. $w_{0}(t)$ is given by the following equation:

$\Delta w_{0}(t)=K_{I} e_{0}(t)-K_{P} \Delta y_{0}(t)-K_{D} \Delta^{2} y_{0}(t)$,

where $e_{0}(t)=\tilde{r}(t)-y_{0}(t) . \quad \tilde{r}(t)$ is derived as follows:

$$
\begin{aligned}
& \tilde{r}(t)=\left[\Delta w_{0}(t)+K_{P} \Delta y_{0}(t)\right. \\
& \left.+K_{I} y_{0}(t)+K_{D} \Delta^{2} y_{0}(t)\right] / K_{I} \text {. }
\end{aligned}
$$

In addition, the user-specified reference model is expressed by the following equation:

$$
\tilde{y}_{m}(t)=\frac{z^{-1} P(1)}{P\left(z^{-1}\right)} \tilde{r}(t),
$$

where $\tilde{y}_{m}(t)$ is reference model output and $P\left(z^{-1}\right)$ is the user-specified polynomial.

\subsection{Off-line learning method in Data-Driven Control scheme by using FRIT}

In this section, an off-line learning method is described by using FRIT. Firstly, the number of neighbors' data $k$ is selected and $\boldsymbol{K}^{\text {old }}(t)$ is calculated by equation (12) by using closed-loop data $w_{0}(t)$ and $y_{0}(t)$. Next, the following steepest descent method is utilized to modify the control parameters:

$$
\begin{aligned}
\boldsymbol{K}^{\text {new }}(t) & =\boldsymbol{K}^{\text {old }}(t)-\boldsymbol{\eta} \frac{\partial J(t+1)}{\partial \boldsymbol{K}(t)} \\
\boldsymbol{\eta} & =\left[\eta_{P}, \eta_{I}, \eta_{D}\right],
\end{aligned}
$$

where $\boldsymbol{\eta}$ denotes the learning ratio and $J(t)$ is defined as the following error criterion:

$$
\begin{gathered}
J(t):=\frac{1}{2} \epsilon(t)^{2} \\
\epsilon(t):=y_{0}(t)-\tilde{y}_{m}(t) .
\end{gathered}
$$

The each partial differential of equation (17) are expanded as follows:

$$
\left.\begin{array}{rl}
\frac{\partial J(t+1)}{\partial K_{P}(t)} & =\frac{\partial J(t+1)}{\partial \tilde{y}_{m}(t+1)} \frac{\partial \tilde{y}_{m}(t+1)}{\partial \tilde{r}(t)} \frac{\partial \tilde{r}(t)}{\partial K_{P}(t)} \\
& =\frac{\epsilon(t+1) P(1) \Delta y_{0}(t)}{K_{I}^{\text {old }}(t)} \\
\frac{\partial J(t+1)}{\partial K_{I}(t)} & =\frac{\partial J(t+1)}{\partial \tilde{y}_{m}(t+1)} \frac{\partial \tilde{y}_{m}(t+1)}{\partial \tilde{r}(t)} \frac{\partial \tilde{r}(t)}{\partial K_{I}(t)} \\
& =\frac{\epsilon(t+1) P(1) \Gamma(t)}{K_{I}^{\text {old }}(t)^{2}} \\
\frac{\partial J(t+1)}{\partial K_{D}(t)} & =\frac{\partial J(t+1)}{\partial \tilde{y}_{m}(t+1)} \frac{\partial \tilde{y}_{m}(t+1)}{\partial \tilde{r}(t)} \frac{\partial \tilde{r}(t)}{\partial K_{D}(t)} \\
& =\frac{\epsilon(t+1) P(1) \Delta^{2} y_{0}(t)}{K_{I}^{\text {old }}(t)}
\end{array}\right\}
$$

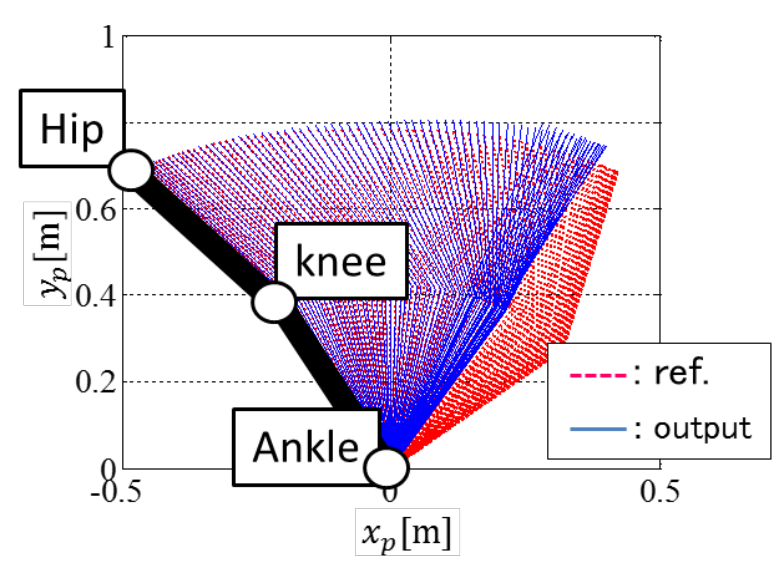

Fig. 4. Walking trajectories by using fixed PID controller as primary controller.
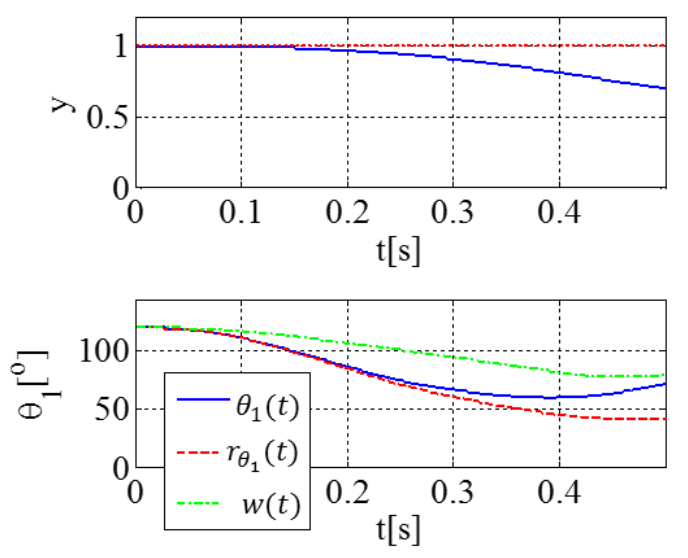

Fig. 5. Trajectories of Kansei signal $y(\mathrm{t})$ and ankle angle $\theta_{1}(\mathrm{t})$ corresponding to Fig. 4.

$$
\begin{gathered}
\Gamma(t)=-\Delta w_{0}(t)-\left\{K_{P}^{\text {old }}(t)+K_{D}^{\text {old }}(t)\right\} y_{0}(t) \\
+\left\{K_{P}^{\text {old }}(t)+2 K_{D}^{\text {old }}(t)\right\} y_{0}(t-1) \\
-K_{D}^{\text {old }}(t) y_{0}(t-2) .
\end{gathered}
$$

Hence, equation (17) and (20) show that control parameters can be learned off-line by using closed-loop data.

\section{Numerical Example}

In this section, the effectiveness of the proposed scheme is verified. The physical parameters ${ }^{5,6}$ in Fig. 2 are set as follows: $I_{1}=0.44\left[\mathrm{~kg} \cdot \mathrm{m}^{2}\right], I_{2}=0.72[\mathrm{~kg}$. $\left.\mathrm{m}^{2}\right], m_{1}=3.26[\mathrm{~kg}], m_{2}=7.00[\mathrm{~kg}], L_{1}=0.42[\mathrm{~m}]$, $L_{2}=0.42[\mathrm{~m}], \quad l_{1}=0.24[\mathrm{~m}], \quad l_{2}=0.24[\mathrm{~m}]$, $g=0.98\left[\mathrm{~m} / \mathrm{s}^{2}\right], \boldsymbol{\eta}=\left[10^{4}, 10^{-3}, 10^{4}\right]$.

Fig. 4 shows the walking trajectories corresponding to Fig. 2 by using a fixed PID controller instead of a primary controller. The dotted red line denotes the reference walking trajectory of brain. Walking support 


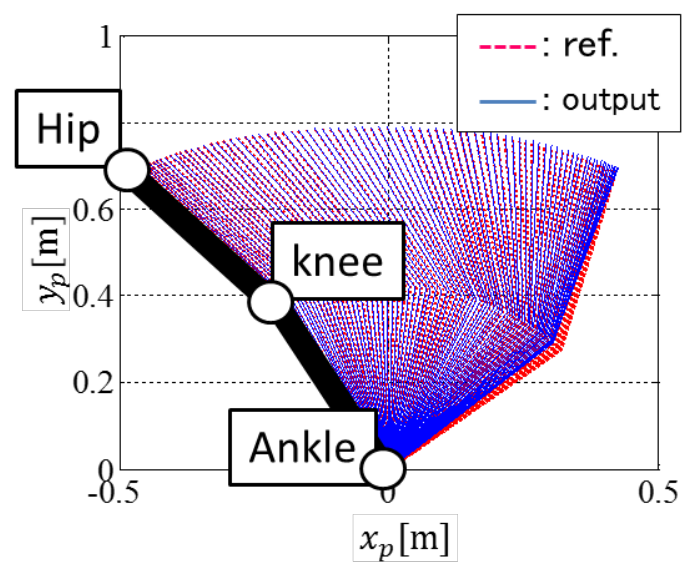

Fig. 6. Walking trajectories by using the proposed scheme.

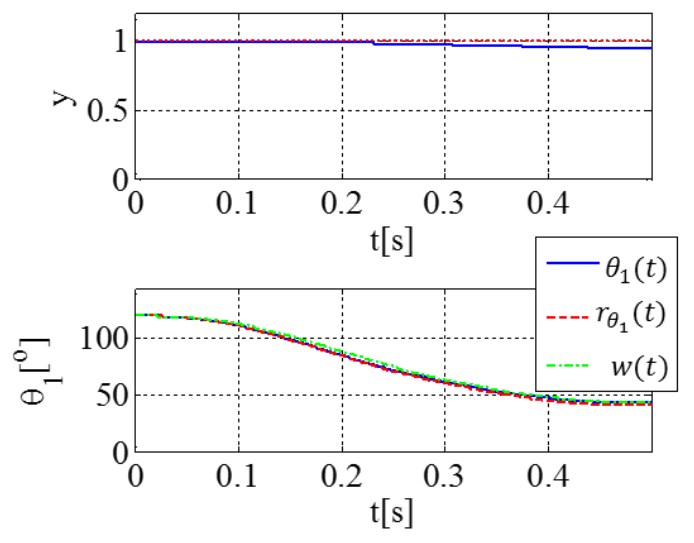

Fig. 7. Trajectories of Kansei signal $y(\mathrm{t})$ and ankle angle $\theta_{1}(\mathrm{t})$ corresponding to Fig. 6.
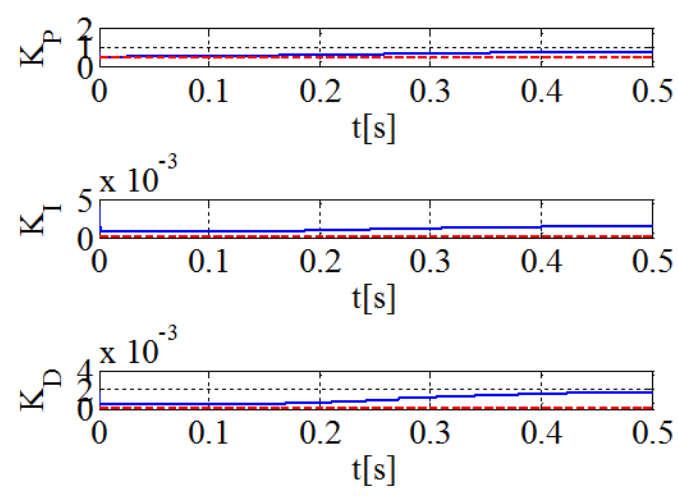

Fig. 8. Trajectories of PID gains corresponding to Fig. 6. does not worked well in Fig. 4 because the blue solid line does not follow the red reference signal. Therefore, the Kansei signal $y(t)$ is not kept around 1 in Fig. 5. Moreover, a fixed PID controller cannot estimate the reference signal well because $w(t)$ does not follow $r_{\theta_{1}}(t)$.

\section{Conclusion}

In contrast, the walking trajectories of Fig. 6 by using the proposed scheme is better than Fig. 7. The effectiveness of the proposed scheme is shown by keeping approximately $y(t)=1$ in Fig. 7. The estimated reference signal $w(\mathrm{t})$ follows $r_{\theta}(\mathrm{t})$ using a data-driven controller, and PID gains in Fig. 8 are adjusted effectively.

In this paper, the field of welfare equipment has been focused and the scheme based on the data-oriented Kansei feedback has been proposed to support each person adaptively. In the proposed scheme, reference signal of a brain was estimated by using a data-driven controller and it supports walking well. The proposed scheme has been verified by numerical example. In the future, experimental result will be considered.

This work was supported by JSPS KAKENHI Grant Number 16K14285.

\section{References}

1. T. Yamamoto and K. Takao and T. Yamada, "Design of a Data-Driven PID Controller", IEEE Trans on control systems Technology, 17(1) (2009) 29-39.

2. S. Yamamoto, "Development of Ankle Foot Orthosis for Hemiplegic Patients Based on Gait Analysis (in Japanese)", The Journal of Japanese Physical Therapy Association, Vol.39, No.4, pp.240-244 (2012)

3. G. T. Fechner, "Elements of Psychophysics", New York: holt, Rinehart and Winston (1966)

4. S. Soma, O. Kaneko and T.Fujii, "A New Approach to Parameter Tuning of Controllers by Using One-Shot Experimental Data: A Proposal of Fictitious Reference Iterative Tuning (in Japanese)", Trans. of the Institute of systems, Control and Information Engineers, 17(2) (2004) 528-536.

5. W. T. Dempster: "Space Requirements of the Seated Operator", WADC(1955)

6. I. P. Herman: "Physics of the Human Body: Biological and Medical Physics, Biomedical Engineering", Springer-Verlag GmbH \& CO. KG(2007) 